\title{
Considering Competition to Solve a Flight Schedule and Aircraft Routing Problem for Small Airlines
}

\author{
J. Díaz-Ramírez*1, Y. Garzón ${ }^{2}$, J.I. Huertas ${ }^{3}$ \\ ${ }^{1}$ Tecnológico de Monterrey \\ Campus Santa Fe, Distrito Federal, México \\ * jenny.diaz@itesm.mx \\ 2,3 Tecnológico de Monterrey \\ Campus Toluca, Toluca, Estado de México, México
}

\begin{abstract}
For the case of low-cost airlines, which are characterized by having a single fleet with a small number of airplanes, in a previous work, a heuristic algorithm (AFS-MRA) was developed to simultaneously find the flight schedule and the aircraft routes subject to maintenance constraints. This work advances this algorithm by incorporating competition in the planning process (MAFS-MRA).

Within a time frame with a given demand data, competition is seen as a game with two players (one airline and all its competitors), where the strategies are all the potential origin-destinations that could be included in the flight schedule, and the payment matrix contains the objective function coefficients that depend on the market share and the routes previously selected.

Numerical experimentation was undertaken using real data for the case of two airlines that operate at Toluca International Airport in Mexico. It was found that, by considering competition, the occupation improves to $3 \%$ and that the number of flights required to satisfy the demand was reduced to $21 \%$. Besides, the updating process reduces the profit computation error in almost $80 \%$, as compared to the real market behavior for the period under study.
\end{abstract}

Keywords: Aircraft maintenance routing, flight schedule design, competition, game

\section{RESUMEN}

Para el caso de aerolíneas de bajo costo, caracterizadas por tener una flota de aviones, en un trabajo previo los autores desarrollaron un algoritmo heurístico (AFS-MRA) para encontrar el programa de vuelos y las rutas de aviones con restricciones de mantenimiento simultáneamente. Este trabajo mejora este algoritmo incorporando los efectos de la competencia en el proceso de planeación (MAFS-MRA).

En un horizonte de tiempo con información de demanda tomada de datos históricos, la competencia se maneja como un juego con dos jugadores (mi aerolínea y todos las demás aerolíneas competidoras), donde las estrategias son todos los potenciales vuelos (orígenes-destinos) que podrían incluirse en el programa de vuelos, y la matriz de pagos contiene los coeficientes de la función objetivo del problema de optimización, los cuales dependen de la participación en el mercado de la aerolínea y de las rutas previamente seleccionadas.

Se desarrolló una experimentación numérica usando datos reales de dos aerolíneas que operan en el Aeropuerto Internacional de Toluca en México. Se encontró que, considerando la competencia, la ocupación mejora en 3\%, el número de vuelos necesarios para satisfacer demanda disminuye en $21 \%$. El proceso de actualización reduce los errores en casi $80 \%$ cuando se comparan con el comportamiento real del mercado en el período bajo estudio.

\section{Introduction}

The air transportation industry has shown a highly variable behavior in the last decades. Between 2001 and 2007, it experienced efficiency improvements yielding high positive incomes but since then, the tendency has been rather negative,

and it is expected that it will not have positive incomes until 2012 [1].

This sector is characterized by high operational costs, and by having an intricate decision-making 
process due to the large number of variables involved. The whole planning process is so complex that it is usually handled sequentially in sub-problems [2, 3]: a) first, the flight schedule is designed; then, b) with the flight schedule (FS) as an input, a fleet assignment problem is solved; c) next, for each fleet, the routing problem is solved; and finally, d) the complex crew management problem is handled. The maintenance constraints of a given fleet are taken into account either during or after the aircraft routing problem is solved $[4,5]$.

On the other hand, the last decade has seen a boom of new small low-cost airlines all around the world. Many of these airlines have just one or two fleets $[6,7]$. This fact eliminates the need for solving the fleet assignment problem, and it also reduces the need for the sequential approach.

Aiming to obtain better global solutions, during the last decade there have been several approaches to solve some of these sub-problems in an integrated fashion. Table 1 summarizes some of them; it can be seen that most of them are related to the last two problems [6].

\begin{tabular}{lccccc}
\hline \multicolumn{1}{c}{ Author } & Year & FS & FA & AR & CM \\
\hline Diaz et al & 2011 & X & & X & \\
Diaz & 2007 & X & & X & X \\
Nitika \& Pal & 2007 & X & X & & \\
Gao \& Johnson & 2007 & & X & & X \\
Papadakos & 2006 & & X & X & X \\
Sandhu \& & 2006 & & X & X & X \\
Klabjan & & & & & \\
Huisman et al & 2004 & & & X & X \\
Klabjan et al & 2002 & & & X & X \\
Stojkovic \& & 2001 & X & & & X \\
Soumis & & & & & \\
Cordeu et al & 2001 & & & X & X \\
Haase et al & 2001 & & & X & X \\
Banhart et al & 1998 & & & X & X \\
Banhart et al & 1998 & & X & & X
\end{tabular}

FS: Flight Schedule, FA: Fleet Assignment, AR: Aircraft Routing, CM: Crew Management

Table 1. Proposals for integration of problems in the airline planning process

\subsection{Motivations}

This work looks for an integrated alternative for the planning process of small scale airlines where the following four typical assumptions are not valid:

- Assumption 1: The best flight schedule design is given. In the sequential planning process it is assumed that the flight schedule used to solve the subsequent problems is the optimal one.

- Assumption 2: Static profit. The objective function coefficients of the optimization problems are known in advance and fixed.

- Assumption 3: Independence of legs. The objective function coefficients in the optimization models (e.g., profit, income, occupancy) are independent.

- Assumption 4: Competition absence. Since the flight schedule is usually fixed and given to solve fleeting and routing problems, competition among flights is usually not considered and therefore "all of the flights must be covered".

\subsection{Background: The AFS-MRA}

An approach to solve the aircraft single-fleet flight schedule design problem together with the routing problem with maintenance constraints was proposed in [6]. The formulation is based on a connected graph $G=(V, A)$ where the nodes in $V$ represent the legs of a timetable, and the $\operatorname{arcs}$ in $A$ represent only legal connections between compatible nodes. Each leg of the network is a candidate to be included in the final flight schedule.

The AFS-MRA (Airline flight scheduling and maintenance routing algorithm) proposed to solve this problem is summarized in Appendix 1. It consists of a greedy heuristic that starts from a given large number of potential flight legs considered by the marketing division, then it identifies at each cycle the route that provides the maximum profit, defining simultaneously both the flight schedule and the aircraft routing, considering at the same time maintenance constraints and fleet 
size. These profits are assumed to be given and fixed for each flight with a specific departure time. Each route is found by solving a model called AMRP, which is a simple routing model with side constraints (i.e., maintenance contraints). See Appendix 2 for further details of the AMRP model. Numerical experiments showed that this algorithm produce good results [6].

The AFS-MRA was designed to eliminate assumption 1. Since it is a greedy algorithm, it is possible to update the objective function coefficients each iteration and therefore eliminate assumption 2. Assumptions 3 and 4 are removed by accepting the strong relationship among FS profits, market demand and competitors' FS.

With these considerations in mind, the objective of this work is to improve the AFS-MRA by incorporating a dynamic profit coefficient function that updates the potential legs remaining in the network every time a new route is found, through a function that estimates the competition effects.

\section{Dynamic approach to consider competition}

We consider that there exists competition when, for a given time window, the demand is less than the market offers. Game theory is the study of the strategic behavior of two or more individuals interacting, when each individual decision comes from what each one expects from the other's decision [8]. That is, it considers the interaction among individuals. For this reason, we rest on this discipline to define the profit function.

\subsection{The game}

A game is defined through three elements: players, strategies and a payment matrix. The game considered here has 2 players. Notice that the players could be defined as the airline of interest (my airline), and the others (the competition) as the second player. Each player competes in a given origin-destination (OD). The strategies, or the set of finite options that each player has are the departure times of all of the possible flights to be included in the flight schedule. Finally, the payment matrix contains the payment (or profit) associated to each strategy for each player. This payment is computed by grouping the multiple options in time windows where the demand is assumed to be homogeneous and constant.

According to the basic classification of the games [ 8 - 10], this game is: a) non cooperative, by law, given that both players offer flights in the same $\mathrm{OD}$; b) a sum zero game, which means that the benefit for one airline means a loss for the other; c) dynamic, since the game is played several times for a given horizon of planning; and d) of complete and symmetric information, since each player knows the payment matrix, and has access to historic information from previous itineraries.

These characteristics allow us to consider just the payment for one of the players (my airline). One game is defined for each $O D$ and for each day of the week because demand varies within-week.

\subsection{Strategy definition: Time windows}

A time window $w$ is defined as the interval of time in which the demand $d(w)$ is shared and uniform for every departing leg. Historical data from airlines in Mexico show that demand changes according to the day of the week. Figure 1 shows, as an example, historical demand data for Monday departing legs TolucaMonterrey, from all the commercial airlines, measured as percentage of occupancy during summer 2009 [11].

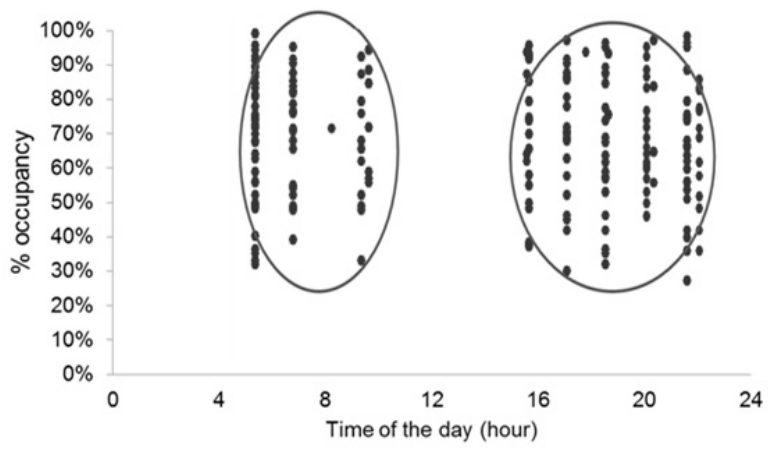

Figure 1. Occupancy percentage of TolucaMonterrey legs during every Monday, summer 2008.

Aiming to identify time windows, cluster analysis was applied over the dyads $\left(s t_{k}, o_{k}\right)$, where $s t_{k}$ is the departure time of leg $k$, and $o_{k}$ is its actual occupation percentage [12 - 14]. 
Two different procedures were applied to check the sensitivity of the results on the technique used to define the time windows. The first one is a bottomup hierarchical technique using the Calinski index [15] to determine the number of clusters (windows). This index considers the sum of squares within and between the clusters. It produces a high number of clusters. For example, with available demand data from Jan-Jun 2008, a day was divided in 12 to 30 clusters, giving a total of 1,960 time windows for a set of 14 ODs. The second technique was the Kmeans. A different $K$ was defined for each $O D$, considering that a time window should not be wider enough to allow two legs in the same window to be selected in the same route. In this case, a day was divided into 3 to 5 clusters, giving a total of 403 time windows. This option provides a more reasonable number of clusters.

Once the historical legs were grouped into clusters, the day was partitioned giving equal distance between the last leg in one cluster and the first leg in the consecutive cluster as it is shown in Figure 1. The demand for each cluster was defined as the average aggregated demand (i.e., market's OD demand for all airlines with the same OD) of the historical data within that cluster.

\subsection{The AFS-MRA considering competition}

In the original AFS-MRA, all routes are defined in one closed cycle. In the modified AFS-MRA proposed here, the profit coefficients are updated before the routing process starts, and those coefficients are calculated as a function of the strategy of the competitors (flight schedule). Next, one route is generated at a time, and based on the output (the route just found), the profit coefficients are updated again depending on whether the demand of the corresponding time window has been covered or partially covered. This process is sketched in Figure 2. Notice that the AMRP model remains the same.

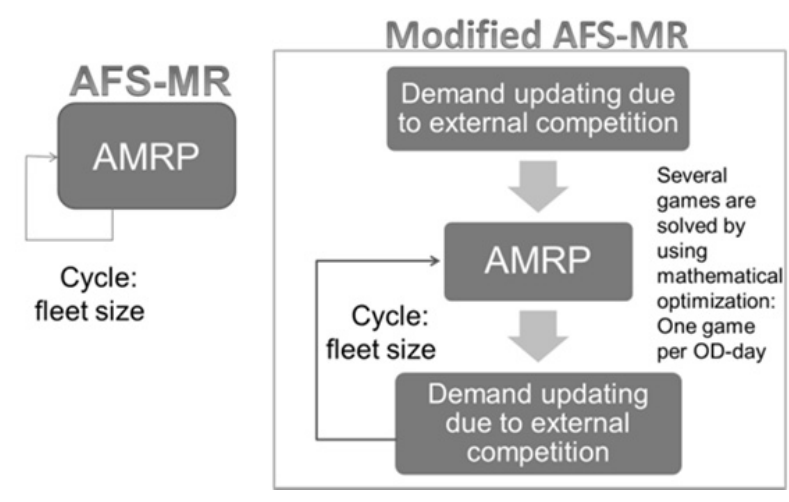

Figure 2. llustration of the original vs. modified AFS-MRA.

The profit function can be considered as a linear combination of the profit given by the demand of leg $\mathrm{i}, d_{i}$, the distance of leg $\mathrm{i}, l_{i}$, price differentiation of leg $\mathrm{i}, c_{i}$, the deadhead between two consecutive flights in a route, $t_{i j}$, and so on. That is $p_{i j}=k_{1} d_{i}+k_{2} l_{i}+k_{3} c_{i}+k_{4} t_{i j} \ldots$ In this work we considered only the updating of the profit given by the demand $d_{i}$.

The modified algorithm is as follows:

Input: potential flights, depots, profit coefficients of potential flight legs, time windows for each OD each day of the week, $w$, time windows' demand: $d(w)$, number of flights offered by competitors in time window $w, C(w)$, number of flights offered by the airline in time window $w, I(w)$, total number of flights offered in time window $w, T(w)$, number of flights selected by a route in window $w, R(w)$, fleet size, $F$, iteration or route number, $t$, demand of flight $i$ if it is going to be the $R(w)+1$-th flight selected in window $\mathrm{w}, d_{i r}$, upper bounds of $y_{i j}: U_{i j}=1 v_{i w}=1$ if departure time of leg $i \in$ time window $w, v_{i w}=0$ otherwise. Note that $T(w)=C(w)+R(w)$ 
Network construction

Updating of profit coefficients of potential flight legs:

$$
\begin{gathered}
t=0 ; R(w)=0 \\
\forall w: T(w) \leftarrow C(w) \\
\forall i: v_{i w}=1 \quad d_{i 0}=\frac{d(w)}{\left(C(w) v_{i w}\right)+1}
\end{gathered}
$$

While $t<F$, not all potential legs have been selected, and the previous route was feasible:

Solve AMRP model. Finds $y_{i j}=1$

Update the network: $\forall i \in N$, if $y_{i j}=1$,

$U_{i j} \leftarrow 0 \quad j:(i, j) \in E$

Updating of profit coefficients of potential flight legs:

$$
\begin{aligned}
& \forall w, \forall i \in N: y_{i j}=1 \text { and if } v_{i w} y_{i j}=1, T(w) \leftarrow T(w)+1 \\
& R(w) \leftarrow R(w)+1 \\
& t=t+1 \quad d_{i, R(w)}=\frac{C(w) d(w)}{T(w)(T(w)+1)}
\end{aligned}
$$

End while

Output: final flight schedule and routes satisfying maintenance constraints, estimated total covered occupancy percentage.

\subsection{Dynamic payment matrix updating}

The modified algorithm considers two updating processes. The first one is done before the routing process and considers the external competition. The second one is done immediately after each route is found, and it considers the internal competition. This recognizes that flights to be selected in future routes, belonging to time windows in which other flights have been selected in previous routes, are mutual competitors. Considering this, the updating process identifies the added demand that would be covered by an additional flight in the same time window.

\subsubsection{Demand updating due to external competition}

Eq. 1 updates the estimated demand to be covered by a flight in a time window where there exist other competitors' offers, under the assumption of equal market power. In this way, the demand associated to each possible flight corresponds to the market participation that such flight would have, given that other flights are already in the market for the same time windows. These $d_{i 0}$ are used as the objective function coefficients in the AMRP model the first time it is solved.

Without the equal market power assumption, Eq. 1 can be generalized as Eq. 3, where $a$ and $b$ represent the competition and the airline market power, respectively.

$$
d_{i 0}=\left(\frac{d(w)}{a C(w) v_{i w}+b}\right) b
$$

Where $a, b$ satisfy $a+b=1$

\subsubsection{Demand updating due to internal competition}

Once a route is found, Eq. 2 updates the estimated demand to be covered by additional flights to be flown in a time window where there are already $R(w)$ flights selected in previous routes. In this way, $d_{i, R(w)}$ reflects only the added demand that flight $i$ will get for the airline. In other words, $d_{i, R(w)}$ is the additional market participation that the new flight will give to the airline, given the other flights offered in the same time window, they are either offered by competitors or by the airline itself. Every time $R(w)$ increases, these $d_{i, R(w)}$ are updated and they are used as the objective function coefficients in the AMRP model to be solved at the subsequent iterations of the algorithm.

The derivation of Eq. 2 that helps to understand the updating process is presented in Appendix 3.

\section{Numerical Experiments}

\subsection{Conditions and assumptions}

In order to demonstrate the methodology proposed in this work, the AFS-MRA was modified considering the following specifications:

Game conditions: a) Players: Airline 1 and Airline 2 , both covering the demand in each origindestination being considered; b) the problem is 
solved for Airline 1. Second airline's flights are considered as external competition; c) the flight options for every OD are grouped into finite time windows.

Assumptions: a) the demand in a given time window is homogeneous; b) the interaction among flights is a function of their departure time.

Data conditions: a) the demand considered comes from the market, not just from one airline; b) demand varies depending on the day of the week. This forces us to define time windows for each OD, for each day of the week; c) the objective function coefficients consider the profit as a function of the occupation percentage of leg $i$ only, this is, $p_{i j}=p_{i}=$ $\%$ occupancy $(i)$. More components such as distance and price differentiation (e.g., classes) should be considered in further stages to make a more robust profit objective function; and finally, d) the time windows and the demands were taken for each OD as the same ones as their inverse. (i.e., demands and time windows for $A-B$ are the same that $B-A)$.

\subsection{Real data used for validation}

Currently, there are two companies which have their hubs at International Airport of Toluca, Mexico. They were the only airlines with commercial flights operating in this airport during 2008. Both airlines initiated operations in the second quarter of 2006, operate with only one fleet whose capacity is approximately the same (144 vs. 150 seats), their fleet is approximately the same size (20-23 airplanes), and share at least 9 domestic ODs. These circumstances create a particular market situation, where it can be said that they have equal market power and that the differences in the market participation in some destinations are due to marketing conditions, not to operational capacities (e.g. 44\%-56\% departing from Toluca in 2007). Data to compute total market demand was taken from [16-19].

Real data from January to June 2008 for these two airlines was used to demonstrate the dynamic updating process of the objective function coefficients. Four ODs shared by both airlines (round trip) were selected, potentials flights were considered with a 10 minutes difference (for each
$\mathrm{OD}$ and for each day of the week) giving a total of 5,370 potential flight legs.

The following computational packages were used: Stata 10.1 to identify clusters, Matlab 7.6, Java 1.6 and GAMS/CPLEX 22.3 to solve the mathematical model.

\subsection{Metrics of performance}

The assumption 3 in section 0 in the original method promotes an over estimation of the demand to be covered. To measure the improvement of the proposed methodology in this regard, it was defined an oversupply metric. On the other hand, to measure the demand satisfaction, the differences between the demand and the offer for every flight were evaluated and they are reported as the sum of square differences (SSD). These metrics are defined as follows:

Oversupply: it measures the average percentage of occupation of the flights. An increment in this value in the dynamic approach will give us evidence of a reduction of the extra supply to cover the same total weekly demand. It is also measured as the total number of flights in excess or "empty". This is an aggregate measure that summarizes the total empty seats of the flight schedule.

Sum of square differences ( $S S D$ ): taking the differences between demand's and supply's occupation percentage, SSD is the sum of the square differences of every covered flight by every approach. SSD allows us to verify the hypothesis that the modification proposed in this research will generate routes whose offer will be closer to the real demand. Other operational measures as cost, for example, were already checked in the original approach.

\subsection{Results}

Seven routes covering a total of 98 flight legs were produced. Given the market conditions and the circumstances of single fleet with the same capacity, demands can be expressed in a single unit: number of (full) flights. 0 presents the oversupply offered to cover the total weekly demand and the average occupation according to each approach. It also presents the SSD of the 
supply with respect to the real demand for both approaches.

\begin{tabular}{lccc}
\hline & AFS-MR & DAFS-MR & $\begin{array}{c}\text { Improvement } \\
(\%)\end{array}$ \\
\hline $\begin{array}{l}\text { Oversup } \\
\text { ply } \\
\text { (number } \\
\text { of flights) } \\
\%\end{array}$ & 14,55 & 11,47 & 21.2 \\
$\begin{array}{l}\text { average } \\
\text { occupati } \\
\text { on }\end{array}$ & 85.15 & 88.3 & 3.14 \\
$\begin{array}{l}\text { Sum of } \\
\text { square } \\
\text { differenc } \\
\text { es (SSD) }\end{array}$ & 12.65 & 2.57 & \\
\hline
\end{tabular}

Table 2. Aggregated results AFS-MR vs. AFS-MR Modified

Results show that by considering competition the occupation improves in 3\%, 21\% less flights are offered, and the updating process reduces the profit computation error in almost $80 \%$, as compared to the real market behavior of the period under study.

\section{Conclusions}

Many low-cost airlines are characterized by having a single fleet of small number of airplanes. This fact eliminates the need of solving the fleet assignment problem and considerably simplifies the planning process, reducing the need of a sequential approach in such process and therefore some of the sub-problems can be solved in an integrated fashion.

In a previous work, a heuristic methodology was developed to find simultaneously the flight schedule and the aircraft routes that respect maintenance constraints. This work advances that methodology by incorporating competition in the planning process.

Within a time frame with a historically given demand, the competition is seen as a game with two players (my airline and all the competitors), where the strategies are all the legs that could be included in the flight schedule, and the payment matrix is the objective function that includes coefficients that depend on the market share.
The heuristic methodology proposed to find simultaneously flight schedule and aircraft routes is a greedy algorithm (AFS-MRA) that at each cycle find the most profitable route. The coefficients of the objective profit function are updated every time a decision is made. Furthermore, the updating process considers the possibility of offering an additional identical route within the same time frame but with the resulting "added profit" that takes into consideration that the route was already selected (offered). The AFS-MRA finishes when there is no other profitable route left or when the fleet is $100 \%$ scheduled.

To measure the improvement of the AFS-MRA, two metrics were established: The oversupply which is measured with respect to the occupation percentage and the total number of flights, and the sum of square differences (SSD) which measures the differences with respect to the actual demand over the period under study.

Numerical experimentation was undertaken using real data from January to June of 2008 for the case of two airlines that operate in Toluca International Airport in Mexico. Results show that, by considering competition, the occupation improves in 3\%, 21\% fewer flights are offered, and the total profit increases by almost $80 \%$.

\section{Appendix 1. AFS-MR Algorithm}

Input: potential flights, depots, profit coefficients of potential flight legs, $\mathrm{F}=$ fleet size, upper bounds of $y_{i j}: U_{i j}=1$

\section{Network construction}

nroutes $=0$

AMRP

While (nroutes $<F$, not all potential legs have been selected, and previous route was feasible) do:

- Solve AMRP model. Identify $y_{i j}=1$

- nroutes $=$ nroutes +1

-Update the network

$$
\left(\forall i \in N: y_{i j}=1, \quad U_{i j} \leftarrow 0 \text { for all } j:(i, j) \in E\right)
$$


Output: final flight schedule design and routes satisfying maintenance constraints.

\section{Appendix 2. AMRP Model}

The AMRP model is a VSP with side constraints, with decision variables defined as

$$
y_{i j}=\left\{\begin{array}{l}
1 \text { if leg } j \text { is covered inmediately after leg } i \\
0 \text { otherwise }
\end{array}\right.
$$

Profit coefficients $p_{i j}$ that can depend both on leg $i$ and $\operatorname{arc}(i, j)$.

$$
\begin{gathered}
z_{A M R P}=\max \sum_{(i, j) \in A} p_{i j} y_{i j} \\
\text { Subject to: } \\
\sum_{j:(i, j) \in A} y_{i j}-\sum_{j:(j, i) \in A} y_{j i}=0 \quad \forall i \in N \cup \Delta \cup \Delta^{\prime} \\
\sum_{s(d):(s,(d)) \in \Lambda} y_{s, s(d)}=1 \quad \forall s^{d} \in \Delta \\
\sum_{t(d):(t(d), t) \in \Lambda} y_{t(d), t}=1 \quad \forall t^{d} \in \Delta^{\prime} \\
\sum_{v} \theta_{u, v} * \sum_{(i, j) \in E_{v}^{B}} y_{i j} \geq 1 \quad \forall u \\
y_{i j} \leq U_{i j}, \text { integer } \quad \forall(i, j) \in A
\end{gathered}
$$

Where, a depot $d=1, \ldots|\Delta|$ is an arrival or departure location where a plane is allowed to stay overnight. $\Delta=\{s(d), \forall d\}$ and $\Delta^{\prime}=\{t(d), \forall d\}$ are the set of depot nodes where an aircraft route can start and end, respectively. $d=1$ represents the maintenance base.

$\Lambda=\bigcup_{\forall d}\{(s, s(d)),(t(d), t)\}$ is the set of arcs connecting depot and end-depot nodes with a source node $s$ and a sink node $t$, respectively, and $E_{v}^{B}$ is the set of overnight arcs at base during night. Let $u$ and $v$ be two day-index sets, where $u$ refers to the day to which each maintenance constraint applies, and $v$ refers to the elements of each constraint.

The maintenance constraints [8] say that any aircraft route must spend at least one night at the base every nightsout +1 consecutive days, where $\Theta=\left\{\theta_{u, v}\right\}$ is the $(7 \times 7)$ coefficient matrix in Eq. 8, where $\theta_{u v}=1$ if overnight arcs on day $v$ are considered for the constraint of the day $u$, and $\theta_{u v}=0$ otherwise.

\section{Appendix 3. Proof equation 0}

This will be done by induction, for a given time window $w$. We have the total demand $d(w), T(w)$ is the total current flights offered (by competitors and my airline) in the window, $R(w)$ is the number of routes chosen that have a flight in the time window $w$. We will call just $d_{R}$ to $d_{i, R(w)}$. An updating is done when at any iteration, $y_{i j}=1$ and $v_{i w}=1$.

$R(w)=0, T(w)=C(w)$ We have by Eq. 0 that $d_{0}=d(w / C(w)+1$.

$$
\text { For } C(w)=1, d_{0}=d(w) / 1+1=(1 / 2) d(w)
$$

After a route is found, and $\exists i: y_{i j}=1 \wedge v_{i w}=1$, then for any $w$ :

$R(w)=R(w)+1=1, T(w)=C(w)+1$.

For $C(w)=1$, then $R(w)=1, T(w)=2$, and $d_{1}=d(w) /(2 \cdot 3)=(1 / 6) d(w)$

With $C(w)=1$ this means that if a third flight is offered in the same time window, and using the equal market power, two flights offered by the airline will go for $2 \times d(w) / 3$. But in the first route, the airline is already considering that the first flight goes for $d(w) / 2$, which means the second flight will go for an additional $2 d(w) / 3-d(w) / 2=d(w) / 6$. See Figure 3 . 
After a second route is found such that $\exists i: y_{i j}=1 \wedge v_{i w}=1$, then for $C(w)=1$, $T(w)=T(w)+1=3$ and $d_{2}=d(w) /(3 \cdot 4)=(1 / 12) d(w)$
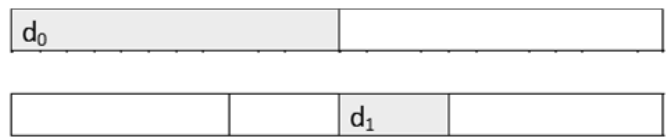

\begin{tabular}{|l|l|l|l|l|}
\hline & & & $\mathrm{d}_{2}$ & \\
\hline
\end{tabular}

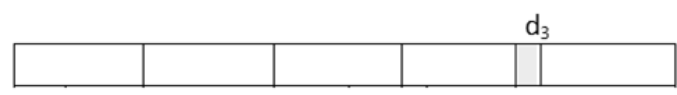

Figure 3. Updating demands due to internal competition. Scheme when $C(w)=1$.

Again, if four flights are offered in the same time window, the three from the airline would go for $(3 / 4) d(w)$, and the fourth one will go for $3 d(w) / 4-d(w) / 2-d(w) / 6=d(w) / 12$

For $R(w)=3$, and if $\exists i: y_{i j}=1 \wedge v_{i w}=1$, then $T(w)=4$ and

$d_{3}=d(w) / 4(5)=(1 / 20) d(w)=(1 / 5) d_{2}$

or $(4 / 5-1 / 2-1 / 6-1 / 12) d(w)=d(w) / 20$

Getting rid of the $(w)$, let the induction hypothesis be $d_{R-I}=C d /((T-1) T)$, which is the demand for flight $i$ : $v_{i w}=1$, in the $R$-th route to be chosen with a flight in $w$.

We want to prove that if the $(R+1)$-th route is found (with a flight $i: v_{i w}=1$ ), or in other words, if the $(R+1)$-th flight in the window is selected, it will have demand: $d_{1}=C d /(T(T+1))$.

Recall $T=R+C$, then $d_{R-1}=C d /(T-1) T=C d /(R+C-1)(R+C)$

So far, the $R$ flights selected in the window cover $(R / T)$ of the demand, and $(R+1)$ flights will go for $[(R+1) /(T+1)]$ of the demand. So the added

$$
\begin{aligned}
d_{R} & =((R+1) /(T+1)) d-(R / T) d \\
& =((R+1) /(R+C+1)-R /(R+C)) d \\
& =(((R+1)(R+C)-R(R+C+1)) / T(T+1)) d \\
& =(((R+C)(R+1-R)-R)) /(T(T+1)) d \\
& =C d /(T(T+1))
\end{aligned}
$$

demand of the $(R+1)$-th flight is given by the difference of these values.

\section{References}

[1] International Air Transport Association. IATA Economic Briefing: The impact of recession on air traffic volumes. 2008.

[2] D. Klabjan. "Large Scale Models in the Airline Industry". In G. Desaulniers et al. (eds), Column Generation. Springer US, 2005, pp 163-195.

[3] D. Klabjan, et al. "Solving Large Airline Crew Scheduling problem: Random Pairing Generation and Strong Branching". Computatiional Optimization and Algorithms, vol. 20, pp. 73-91, 2001

[4] C. Barnhart, and A. Cohn. "Airline Scheduling Problems: Accomplishments and opportunities", Manufacturing \& Service Operations Management, vol. 6, no. 1,2004

[5] C. Barnhart et al. "Flight Strings Models for Aircraft Fleeting and Routing", Transportation Science, vol. 32, no. 3, 1998.

[6] J. Díaz. "An Integrated approach for the single-fleet aircraft and crew scheduling problems". Toluca : Ph.D. Thesis, Tecnologico de Monterrey Campus Toluca, Toluca, 2007.

[7] flylowcostairlines. flylowcostairlines. [Online] 2011. [retrieved: February 8, 2011.] www.flylowcostairlines.org.

[8] S. Monsalve. "John Nash and Game Theory". Original Language: Spanish, Bogota : Universidad Nacional de Colombia, Lecturas Matemáticas, vol. 24, pp. 137-149, 2003.

[9] H. Kuhn. "Lectures on the Theory of Games", Princeton University Press IX. 2003

[10] F. Vega-Redondo. "Economics and the Theory of Games". Cambridge: Cambridge University Press, 2003. 
[11] Secretaria de Comunicaciones y Transporte. [Online] Statistics. www.sct.gob.mx. Original Language: Spanish.

[12] C. Sugar and G. M. James. "Finding the Number of Clusters in a Dataset: An Information-Theoritec Approach". Journal of the American Statistical Association, no. 463, pp. 750-763, 2003, DOI: 10.1198/016214503000000666.

[13] D. Tasoulis and M. Vrahatis. "Generalizing the kWindows Clustering Algorithm in Metric Spaces", Mathematical and Computer Modellling, 2007.

[14] X-T. Zhang et al. "A model-based clustering for time series with irregular interval". Proceedings of the Third International Conference on Machine Learning and Cybernetics, vol. 5, pp. 2883 - 2888. 2004. DOI: 10.1109/ICMLC.2004. 1378524 .

[15] T. Calinski and J. Harabasz. "A dendrite method for cluster analysis". Communications in Statistics, vol. 3, no. 1, pp. 1-27, 1974.

[16] Dirección Nacional de Aeronáutica. "Monthly Statistical Report by Origin-Destination 2008". Original Language: Spanish: http://dgac.sct.gob.mx/ Retrieved on March 19th, 2008.

[17] International Air Transport Association. "IATA Annual Report". 2008.

[18] International Air Transport Association IATA. "IATA Economic Briefing Airline Profitability 2006, 2007.

[19] Secretaría de Desarrollo Económico. Gobierno del Estado de México. [Online] GDP Indicators in the State of Mexico, $=$ Mexico. $=,=2007$.

http://www1.edomexico.gob.mx/sedeco/. Original

Language: Spanish. 